\title{
Statistical approaches in landscape genetics: an evaluation of methods for linking landscape and genetic data
}

\author{
Niko Balkenhol, Lisette P. Waits and Raymond J. Dezzani \\ N. Balkenhol (nbalkenhol@vandals.uidaho.edu) and L. P. Waits Dept of Fish and Wildlife Resources, Univ. of Idaho, Moscow, ID \\ 83844-1136, USA. - R. J. Dezzani, Dept of Geography, Univ. of Idaho, Moscow, ID 83844-3021, USA.
}

\begin{abstract}
The goal of landscape genetics is to detect and explain landscape effects on genetic diversity and structure. Despite the increasing popularity of landscape genetic approaches, the statistical methods for linking genetic and landscape data remain largely untested. This lack of method evaluation makes it difficult to compare studies utilizing different statistics, and compromises the future development and application of the field. To investigate the suitability and comparability of various statistical approaches used in landscape genetics, we simulated data sets corresponding to five landscape-genetic scenarios. We then analyzed these data with eleven methods, and compared the methods based on their statistical power, type-1 error rates, and their overall ability to lead researchers to accurate conclusions about landscape-genetic relationships. Results suggest that some of the most commonly applied techniques (e.g. Mantel and partial Mantel tests) have high type-1 error rates, and that multivariate, non-linear methods are better suited for landscape genetic data analysis. Furthermore, different methods generally show only moderate levels of agreement. Thus, analyzing a data set with only one method could yield method-dependent results, potentially leading to erroneous conclusions. Based on these findings, we give recommendations for choosing optimal combinations of statistical methods, and identify future research needs for landscape genetic data analyses.
\end{abstract}

Landscape genetics is an emerging interdisciplinary research area that combines population genetics, landscape ecology, and spatial statistics (Manel et al. 2003, Storfer et al. 2007). The goal of landscape genetics is to describe and explain how landscape attributes affect genetic variation of plant and animal populations. Landscape genetic approaches have the potential to greatly enhance our knowledge of how landscape heterogeneity influences genetic population structure, gene flow, and adaptation (Holderegger and Wagner 2008). Results from landscape genetic studies are also increasingly used to address questions related to species management and conservation (Epps et al. 2007). Because of the immense potential for theoretical and applied contributions, the number of landscape genetic studies is growing rapidly (Holderegger and Wagner 2006). At the same time, the statistical approaches used to infer landscape-genetic relationships remain largely untested.

Landscape genetic research usually involves two analytical steps (Manel et al. 2003) that can be combined within a single analysis. In the first step, patterns of genetic variation are described, and in the second step, these patterns are statistically correlated with environmental (e.g. landscape) variables. Thus, the final goal of landscape genetics is the quantification of landscape effects on genetic variation (Storfer et al. 2007). In the last few years, a multitude of statistical approaches have been proposed for this second step of landscape genetics. These methods range from relatively simple correlation statistics to more advanced and complex Bayesian modeling approaches. Furthermore, different methods require different input formats for the landscape data, which can be either pairwise (e.g. landscape resistance measured between all sample locations; Adriaensen et al. 2003), or patch-specific (e.g. habitat types or connectivity indices; Moilanen and Nieminen 2002). Additional research is greatly needed to determine whether all of the proposed methods produce accurate, valid, and repeatable results in a landscape genetic context.

Researchers interested in landscape genetics are faced with a large variety of analysis options with no current guidelines or recommendations on choosing appropriate methods. A better understanding of the advantages and limitations of current analytical approaches is needed to develop a theoretical framework for landscape genetics, improve the accuracy of interpretations, and allow comparisons across studies. Furthermore, evaluating existing statistical approaches is crucial to the long-term development of landscape genetics as it is the first step for developing new and improved analysis approaches.

Here, we present the first evaluation of analytical approaches currently used for the second step of landscape genetics. We evaluate eleven statistical techniques through 
the analysis of simulated data with known landscape-genetic relationships, and compare methods based on their statistical power, type-1 error rates, and overall success rates. Specifically, our goals are to: 1) compare the utility of different methods available for analyzing landscape genetic data; 2) develop recommendations for choosing an optimal set of methods for analyzing empirical data sets; and 3) identify remaining challenges and future research needs related to landscape genetic data analysis.

\section{Methods}

\section{Landscape-genetic simulations}

Evaluating the performance of different methods currently used in landscape genetics requires the use of data sets with known landscape-genetic relationships. Simulations are ideal for this purpose, because they can establish a direct (i.e. nonconfounding) relationship between landscape variables and simulated genetic data. However, software for simulating such landscape genetic data currently does not exist, making the simulation approach challenging. Therefore, to create data with non-ambiguous landscape-genetic relationships, we obtained landscape data from a Geographic Information System (GIS) to derive input parameters for an existing population genetic simulation program. For this simulation approach, we identified five landscape genetic scenarios commonly reported in the literature, and for each scenario determined the expected statistical relationship between landscape and genetic data (Table 1). We then used these expected statistical relationships for genetic simulations in Easypop 2.01 (Balloux 2002), an individual-based simulation program in which multi-locus genotypes can be simulated forward in time and under a large variety of conditions (i.e. varying mating schemes, mutation rates, migration models etc.).

Scenario A - null: in this scenario, gene flow is not influenced by any spatial or landscape variables. Genetic structure can arise independently from the landscape, for example due to mutation, drift, or non-random mating schemes (Wright 1977). Also, the landscape data used in a study may not encompass those landscape features that actually influence gene flow, so that no significant correlations should be detected. To simulate such a null scenario, we used a simple island model of migration, in which gene flow does not depend on any spatial or landscape parameters.

Table 1. Description of simulated scenarios, including expected statistical relationship between landscape and genetic data.

\begin{tabular}{|c|c|c|c|c|}
\hline Scenario & $\begin{array}{l}\text { Description and } \\
\text { ecological } \\
\text { justification }\end{array}$ & $\begin{array}{l}\text { Statistical } \\
\text { expectation }\end{array}$ & $\begin{array}{c}\text { Easypop } \\
\text { (Balloux 2002) } \\
\text { simulations }\end{array}$ & $\begin{array}{l}\text { Empirical } \\
\text { example }\end{array}$ \\
\hline A - null & $\begin{array}{l}\text { Genetic differentiation among } \\
\text { populations is not influenced } \\
\text { by space or landscape } \\
\text { variables. }\end{array}$ & $\begin{array}{l}\text { Measures of genetic } \\
\text { differentiation should not be } \\
\text { correlated with any spatial or } \\
\text { landscape variables. }\end{array}$ & $\begin{array}{l}\text { Island model with } 12 \\
\text { populations. }\end{array}$ & $\begin{array}{l}\text { Blank et al. } \\
2007\end{array}$ \\
\hline $\begin{array}{l}\text { B - isolation-by- } \\
\text { distance (IBD) only }\end{array}$ & $\begin{array}{l}\text { In species with spatially } \\
\text { limited dispersal, genetic } \\
\text { differentiation is influenced } \\
\text { by spatial distances among } \\
\text { populations, and populations } \\
\text { further apart show stronger } \\
\text { genetic differentiation. }\end{array}$ & $\begin{array}{l}\text { Measures of genetic } \\
\text { differentiation should be } \\
\text { correlated with geographic } \\
\text { distance "B" among } \\
\text { populations. }\end{array}$ & $\begin{array}{l}\text { Spatial migration model, in } \\
\text { which gene flow depends on } \\
\text { mean dispersal distance and } \\
\text { geographic distances (" } \mathrm{B} \text { ") } \\
\text { among populations. }\end{array}$ & $\begin{array}{l}\text { Rose et al. } \\
2006\end{array}$ \\
\hline $\begin{array}{l}\mathrm{C} \text { - landscape } \\
\text { resistance }\end{array}$ & $\begin{array}{l}\text { The number of migrants } \\
\text { exchanged among } \\
\text { populations often depends on } \\
\text { the landscape between them, } \\
\text { with populations separated by } \\
\text { high resistance landscapes } \\
\text { showing greater genetic } \\
\text { differentiation. }\end{array}$ & $\begin{array}{l}\text { Measures of genetic } \\
\text { differentiation should be } \\
\text { correlated with least-cost } \\
\text { distance "C", which is not } \\
\text { correlated with geographic } \\
\text { distance. }\end{array}$ & $\begin{array}{l}\text { Spatial migration model, } \\
\text { with coordinates obtained } \\
\text { from nmMDS of least-cost } \\
\text { distance matrix "C" } \mathrm{C}^{\text {". This }} \\
\text { least-cost distance is not } \\
\text { correlated with geographic } \\
\text { distance. }\end{array}$ & $\begin{array}{l}\text { Cushman et al. } \\
2006\end{array}$ \\
\hline $\begin{array}{l}\mathrm{D} \text { - landscape } \\
\text { resistance and IBD }\end{array}$ & $\begin{array}{l}\text { The number of migrants } \\
\text { exchanged among } \\
\text { populations often depends on } \\
\text { the landscape and distance } \\
\text { between them, with } \\
\text { populations separated by } \\
\text { high resistance landscapes } \\
\text { showing greater genetic } \\
\text { differentiation. }\end{array}$ & $\begin{array}{l}\text { Measures of genetic } \\
\text { differentiation should } \\
\text { be correlated with least-cost } \\
\text { distance " } \mathrm{D} \text { ", which is } \\
\text { correlated with } \\
\text { geographic distance. }\end{array}$ & $\begin{array}{l}\text { Spatial migration model, with } \\
\text { coordinates obtained from } \\
\text { nmMDS of least-cost distance } \\
\text { matrix "D". This effective } \\
\text { distance is correlated with } \\
\text { geographic distance. }\end{array}$ & $\begin{array}{l}\text { Spear et al. } \\
2005\end{array}$ \\
\hline $\begin{array}{c}E \text { - landscape } \\
\text { boundaries }\end{array}$ & $\begin{array}{l}\text { Genetic differentiation among } \\
\text { populations can be caused by } \\
\text { (linear) landscape boundaries } \\
\text { (e.g. roads, changes in habitat } \\
\text { type), with lower migration } \\
\text { rates across boundaries. }\end{array}$ & $\begin{array}{l}\text { Measures of genetic } \\
\text { differentiation should be } \\
\text { correlated with a "boundary } \\
\text { indicator", i.e. a categorical } \\
\text { variable indicating whether } \\
\text { populations are on the same } \\
\text { side of a boundary or not. }\end{array}$ & $\begin{array}{l}\text { Hierarchical island model } \\
\text { with higher migration rates } \\
\text { within archipelagos, and } \\
\text { lower migration rates among } \\
\text { archipelagos separated by } \\
\text { landscape boundaries. The } \\
\text { number of archipelagos } \\
\text { ranged from } 2 \text { to } 5 \text {, } \\
\text { representing } 1 \text { to } 4 \\
\text { boundaries. }\end{array}$ & $\begin{array}{l}\text { Epps et al. } \\
2005\end{array}$ \\
\hline
\end{tabular}


Scenario B - isolation-by-distance (IBD): under isolationby-distance (IBD), populations separated by greater geographic (i.e. straight-line or Euclidean) distances show greater levels of genetic differentiation (Wright 1943). Such a pattern can arise when gene flow (i.e. the exchange of migrants, seed, or pollen) mostly occurs among spatially close populations, for example in species with limited dispersal abilities (Trizio et al. 2005). Statistically, this means that a significant correlation exists between pair-wise geographic distances and measures of genetic differentiation (e.g. $\mathrm{F}_{S T}$; Wright 1943) among populations. Since biotic, species-specific (i.e. landscape-independent) processes are regulating gene flow in this scenario, it represents a "false" gradient (Legendre 1993). This means that external, environmental processes are not responsible for observed genetic patterns. Thus, in simulations of this scenario, gene flow and resulting genetic differentiation should only be influenced by geographic distances among populations. To simulate this scenario, we used the spatial migration model in Easypop, in which the number of migrants exchanged between two populations depends on the separation distance between them.

Scenario C - landscape resistance: in some species, dispersal is not spatially limited, but the landscape matrix between populations influences individual movements, and thus, gene flow (Ricketts 2001). Populations connected by low-resistance landscapes exchange greater number of migrants, resulting in a significant correlation between genetic differentiation and measures of landscape resistance (McRae 2006). When gene flow is independent of space, and only influenced by the landscape matrix ("pure" landscape effect), geographic distances among populations should not be significantly correlated with genetic data. Thus, we based simulations of this scenario on resistance distances that were not correlated with geographic distances. These resistance distances were then used to describe population separation in the spatial migration model of Easypop.

Scenario D - landscape resistance and IBD: oftentimes, space and landscape resistance will simultaneously influence gene flow (Trizio et al. 2005). Furthermore, landscape resistance is often measured as a function of both distance and resistance between population pairs (e.g. least-cost distance or length of least-cost path; Ray 2005). Thus, populations that are far apart in space are often also separated by high resistance distances, making the distinction between purely spatial and true landscape effects particularly challenging in practice (Cushman et al. 2006). Statistically, this scenario is characterized by an interaction between spatial and landscape effects, and gene flow is influenced by a combination of geographic distances and landscape resistances. Thus, to simulate this scenario, we used resistance distances that were correlated with geographic distances, making it possible to statistically differentiate this scenario from scenario $\mathrm{C}$ described above.

Scenario E - landscape boundaries: genetic structure can also be influenced by distinct landscape boundaries, for example changes in habitat types, roads, or rivers (Sacks et al. 2004, Epps et al. 2005). When such boundaries impede individual movement, gene flow can be limited, leading to increased genetic differentiation across boundaries. Thus, genetic differentiation is correlated with the presence or absence of boundaries between populations. This scenario was simulated in Easypop via the hierarchical island model of migration. In this model, migration among populations belonging to the same group of islands or archipelago (i.e. populations not separated by boundaries) is higher than among populations belonging to different groups (i.e. populations separated by landscape boundaries).

The data required to simulate the different scenarios were extracted from real and artificial landscape data as follows: first, we delineated five study areas across the state of Idaho (USA) (see Supplementary material for more information on the study areas). Then, we created locations for twelve populations in each of these areas, and calculated geographic distances among populations belonging to a certain study area (separation distance "B"). In addition, we calculated least-cost distances among population pairs within each study area (separation distance "D"). We obtained the cost grids required for these least-cost analyses by assigning resistance values to habitat types found within the study areas. Habitat types were based on Idaho GAP data, and cost values ranged from 1 to 1000 . Since the goal was to simulate statistical landscape-genetic relationships, the exact compositions and configurations of the landscapes, resolution of the data, or the ecological meaning of the cost values are not important, as long as the required correlations among the different separation distances were met (Table 1).

In addition to this cost grid based on real landscape data, we manually digitized an artificial cost grid for each study area, and used it to calculate a second set of least-cost distances among population pairs (separation distances "C"). The artificial cost grids were constructed so that spatially close populations were separated by high-resistance habitat, while low-resistance habitats connected populations that were spatially far apart. This approach ensured that separation distances $\mathrm{C}$ for a study area were not correlated with Euclidean distances $\mathrm{B}$ in that area, so that the landscape resistance scenario $\mathrm{C}$ could be simulated. All distance calculations were performed in the Arcview extension PATHMATRIX (Ray 2005). Finally, we determined which population pairs were separated by "landscape boundaries" (e.g. rivers, roads, changes in habitat types).

We then used the landscape data obtained from each study area as input parameters for the genetic simulations in Easypop (Table 1). Since the spatial model available in Easypop requires user-input coordinates rather than distances, all distance matrices (i.e. distances B, C, and D) were transformed into two-dimensional coordinates via non-metric multidimensional scaling. Non-metric multidimensional scaling (nmMDS) is an effective way to summarize pair-wise relationships contained in multivariate distance matrices (Borg and Groenen 2005), and was calculated in Systat 11 (Systat Software 2004). Resulting nmMDS coordinates were then used to describe effective separation distances among populations in the Easypop simulations.

We used each parameter set for four independent genetic simulations in Easypop, so that each scenario was created 20 times (i.e. four genetic simulations in each of the five study areas), leading to a total of 100 landscape-genetic data sets. In each simulation, genetic data for 2400 individuals (100 females and 100 males per population) were created for 15 
microsatellite loci following a mixed model of allelic mutation (SSM/KAM option in Easypop). Each simulation was run for 10000 generations to ensure that populations reached migration-drift equilibrium.

The simulation approach was designed to produce data sets with direct and clear landscape-genetic relationships, while controlling for other factors that may impact the analysis of empirical data. Thus, the number of simulated individuals was chosen to be very high, and population sizes, sex-ratios, number of loci, generation times, etc., were held constant across simulations. However, the scenarios were ecologically realistic, and simulations led to a wide range of data sets that have characteristics similar to those found in empirical studies (Results).

\section{Data analysis}

For each simulated population, we calculated expected heterozygosity $\left(\mathrm{H}_{\mathrm{e}}\right)$ in GENEPOP (Raymond and Rousset 1995), and estimated pair-wise $F_{S T}$ values among populations in FSTAT (Goudet 1995). Significance of $\mathrm{F}_{\mathrm{ST}}$ values was determined through 4999 randomizations. Correlations among the various distance matrices (i.e. distances B, C, and D) were assessed through a Mantel test in ZT (Bonnet and Van de Peer 2002) with 999999 permutations.

\section{Methods used to test for landscape-genetic relationships}

We used eleven different statistical approaches to test for landscape-genetic relationships in the data sets. These methods are briefly described below and summarized in Table 2. An in-depth review of the methods is beyond the scope of this paper, and readers are referred to the method references, program manuals, general statistical textbooks, and other review papers (Legendre and Legendre 1998, Legendre 2000, Clarke and Warwick 2001, Manly 2007) for more detailed information. The programs used to perform the statistical tests, including the number of permutations and other relevant program settings are listed in Table 2.

\section{A: methods using pair-wise landscape data}

1) Mantel test: the standard Mantel test (Mantel 1967) is currently the most-commonly applied method in landscape genetics, and uses permutations to assess the significance of the linear correlation coefficient $(\mathrm{r})$ between two pair-wise (dis-)similarity matrices.

2) RELATE: the RELATE procedure proposed by Clarke and Warwick (2001) is a non-linear alterative to the Mantel test, and calculates the rank-correlation between two pair-wise (dis-)similarity matrices, using permutations to assess significance. Even though RELATE has not yet been applied in landscape genetics, we included it in our analyses, because it provides an intuitive, non-linear extension of the Mantel statistic.

3) MRDM: to assess the effects of several landscape variables, multiple regression of distance matrices (MRDM) has been used in landscape genetics (Holzhauer et al. 2006). Such multiple regression approaches can test for effects of all landscape variables simultaneously (Manly 1986), or add significant explanatory variables through a step-wise procedure (Legendre et al. 1994).

4) BIMR: Bayesian inference of immigration rates (BIMR; Faubet and Gaggiotti 2008) is a new method that uses genetic and pair-wise environmental data to estimate recent migration rates among populations, and to identify the environmental factors that likely influence migration. The approach is based on a generalized linear model (GLM), and uses Markov Chain Monte Carlo (MCMC) methods to obtain posterior estimates.

\section{B: methods using location-specific landscape data}

5) GESTE: the software GESTE (genetic structure inference based on genetic and environmental data; Foll and Gaggiotti 2006) implements a hierarchical Bayesian approach to estimate population-specific $\mathrm{F}_{\mathrm{ST}}$ values and to evaluate which local environmental factors likely contributed to observed genetic structures. Similar to BIMR, it is based on a GLM, and uses MCMC's to estimate posterior distributions.

6) dbRDA: distance-based redundancy analysis (dbRDA; Legendre and Anderson 1999, McArdle and Anderson 2001) is a multivariate method that assesses the influence of local environmental factors on values in a linearly dependent (dis-)similarity matrix through permutations. To account for confounding spatial effects, coordinates of sample areas can be incorporated into the model as covariates (partial dbRDA).

7) BIOENV/BEST: the non-parametric BIOENV (or BEST) procedure (Clarke and Ainsworth 1993) calculates a Spearman-rank correlation coefficient between the pair-wise response matrix and multivariate distances derived from the predictor variables (i.e. the landscape variables). The method uses all possible combinations of predictor variables to determine the models that best fit the dependent data, assesses the significance of the single best model, and determines which variables are associated with this model.

8) CCA: canonical correspondence analysis (CCA; ter Braak 1986) has been used to infer environmental impacts on genetic variation (Angers et al. 1999). CCA is a constraint ordination technique that assumes a uni-modal relationship when describing gradients in the first set of variables (i.e. the dependent variable) in terms of the second (explanatory) variable set. For genetic data, populationspecific allele frequencies can be used for the dependent variable set (Angers et al. 1999).

In addition to these eight "full" methods that test for marginal effects, we also applied three partial methods. In these methods, purely spatial effects on genetic differentiation are accounted for before assessing landscape effects. These partial options are often used in landscape genetics to separate true landscape effects from purely spatial effects.

9) Partial Mantel test: in a partial Mantel test (Smouse et al. 1986), the effect of one pair-wise matrix is controlled for (i.e. its effect is "partialled out") before assessing the correlation between the two remaining matrices. Essentially, this is accomplished by subsequently using the residuals of a first standard Mantel test in a second Mantel test.

10) and 11) Partial dbRDA and partial CCA: in a partial dbRDA and partial CCA, coordinates of sampling locations 
Table 2. Description of statistical approaches, software and program settings used to analyze simulated data sets.

\begin{tabular}{|c|c|c|c|c|c|c|c|}
\hline $\begin{array}{l}\text { Statistical } \\
\text { approach }\end{array}$ & $\begin{array}{l}\text { Method } \\
\text { reference }\end{array}$ & Description & Genetic data & Landscape data & $\begin{array}{l}\text { Software used } \\
\text { in this study }\end{array}$ & $\begin{array}{l}\text { Software } \\
\text { settings }\end{array}$ & $\begin{array}{l}\text { Example } \\
\text { application }\end{array}$ \\
\hline $\begin{array}{l}\text { (Partial) } \\
\quad \text { Mantel test }\end{array}$ & $\begin{array}{l}\text { Mantel 1967, } \\
\text { Smouse et al. } \\
1986\end{array}$ & $\begin{array}{l}\text { Permutation-based test for } \\
\text { correlation between two } \\
\text { distance matrices. In a } \\
\text { partial Mantel test, } \\
\text { correlation is assessed } \\
\text { between two distance } \\
\text { matrices, while } \\
\text { controlling for effects of } \\
\text { factors in a third matrix. }\end{array}$ & $\begin{array}{l}\text { Pair-wise measure of } \\
\text { genetic differentiation } \\
\text { (e.g. pair-wise Fst). }\end{array}$ & $\begin{array}{l}\text { Pair-wise measures of } \\
\text { spatial/effective } \\
\text { separation (e.g. } \\
\text { geographic or least-cost). }\end{array}$ & $\begin{array}{l}\text { ZT (Bonnet and } \\
\text { Van der Peer 2002) }\end{array}$ & $\begin{array}{l}999999 \\
\text { permutations }\end{array}$ & $\begin{array}{l}\text { Coulon et al. } \\
2004\end{array}$ \\
\hline RELATE & $\begin{array}{l}\text { Clarke and } \\
\text { Warwick } 2001\end{array}$ & $\begin{array}{l}\text { Test for rank-correlation } \\
\text { between two distance } \\
\text { matrices. }\end{array}$ & $\begin{array}{l}\text { Pair-wise measure of } \\
\text { genetic differentiation } \\
\text { (e.g. pair-wise Fst). }\end{array}$ & $\begin{array}{l}\text { Pair-wise measures of } \\
\text { spatial/effective separation } \\
\text { (e.g. geographic or } \\
\text { least-cost). }\end{array}$ & $\begin{array}{l}\text { PRIMER } 6 \\
\text { (Primer-E 2006) }\end{array}$ & $\begin{array}{l}9999 \\
\text { permutations }\end{array}$ & None, yet \\
\hline $\begin{array}{l}\text { Multiple regression } \\
\text { on distance matrices } \\
\text { (MRDM) }\end{array}$ & $\begin{array}{l}\text { Legendre } \\
\text { et al. } 1994\end{array}$ & $\begin{array}{l}\text { (Stepwise) regression of a } \\
\text { dependent distance matrix } \\
\text { and multiple independent } \\
\text { pair-wise matrices. }\end{array}$ & $\begin{array}{l}\text { Pair-wise measure of } \\
\text { genetic differentiation } \\
\text { (e.g. pair-wise Fst). }\end{array}$ & $\begin{array}{l}\text { Pair-wise measures of } \\
\text { spatial/effective separation } \\
\text { (e.g. geographic or } \\
\text { least-cost). }\end{array}$ & $\begin{array}{l}\text { PERMUTE! } 3.4 \text { alpha } \\
9 \text { (Legendre et al. 1994) }\end{array}$ & $\begin{array}{l}9999 \text { permutations, } \\
\text { forward selection } \\
\text { with } p \text { to enter }<01\end{array}$ & $\begin{array}{l}\text { Holzhauer et al. } \\
2006\end{array}$ \\
\hline $\begin{array}{l}\text { Bayesian inference } \\
\text { of immigration rates } \\
\text { (BIMR) }\end{array}$ & $\begin{array}{l}\text { Faubet and } \\
\text { Gaggiotti } 2008\end{array}$ & $\begin{array}{l}\text { Bayesian model-based } \\
\text { approach that uses MCMC } \\
\text { techniques to estimate } \\
\text { migration rates and } \\
\text { identifies environmental } \\
\text { variables that most likely } \\
\text { influenced observed } \\
\text { genetic patterns. }\end{array}$ & $\begin{array}{l}\text { Number of exchanged } \\
\text { migrants (estimated } \\
\text { from genotype data as } \\
\text { part of the method). }\end{array}$ & $\begin{array}{l}\text { Pair-wise measures of } \\
\text { spatial/effective separation } \\
\text { (e.g. geographic or } \\
\text { least-cost). }\end{array}$ & $\begin{array}{l}\text { BIMR (Faubet and } \\
\text { Gaggiotti 2008) }\end{array}$ & Default settings & None, yet \\
\hline $\begin{array}{l}\text { Genetic structure } \\
\text { inference based on } \\
\text { genetic and } \\
\text { environmental } \\
\text { data (GESTE) }\end{array}$ & $\begin{array}{l}\text { Foll and } \\
\text { Gaggiotti } 2006\end{array}$ & $\begin{array}{l}\text { Bayesian model-based } \\
\text { approach that uses MCMC } \\
\text { techniques to estimate } \\
\text { population-specific } \\
\text { Fsts, and identifies } \\
\text { environmental variables } \\
\text { that most likely influenced } \\
\text { observed genetic patterns. }\end{array}$ & $\begin{array}{l}\text { Population-specific } \\
\text { Fst (estimated from } \\
\text { genotype data as part } \\
\text { of the method). }\end{array}$ & $\begin{array}{l}\text { Point-wise measures } \\
\text { (e.g. coordinates or } \\
\text { connectivity indices). }\end{array}$ & $\begin{array}{l}\text { GESTE v1 (Foll } \\
\text { and Gaggiotti 2006) }\end{array}$ & Default settings & $\begin{array}{l}\text { Leclerc et al. } \\
2008\end{array}$ \\
\hline $\begin{array}{l}\text { (Partial) distance-based } \\
\text { redundancy analysis } \\
\text { (dbRDA) }\end{array}$ & $\begin{array}{l}\text { McArdle and } \\
\text { Anderson } 2001\end{array}$ & $\begin{array}{l}\text { Canonical form of } \\
\text { principal coordinates } \\
\text { analysis; a direct } \\
\text { ordination method used to } \\
\text { explain one dataset } \\
\text { (e.g. genetic structure) by } \\
\text { another dataset (e.g. } \\
\text { landscape data). In the } \\
\text { partial option, coordinates } \\
\text { are fitted as co-variates. }\end{array}$ & $\begin{array}{l}\text { Pair-wise measure of } \\
\text { genetic differentiation } \\
\text { (e.g. pair-wise Fst). }\end{array}$ & $\begin{array}{l}\text { Point-wise measures } \\
\text { (e.g. coordinates or } \\
\text { connectivity indices). }\end{array}$ & $\begin{array}{l}\text { DISTLM v5 } \\
\text { (Anderson 2004) }\end{array}$ & $\begin{array}{l}9999 \text { unrestricted } \\
\text { permutations under } \\
\text { the regression model }\end{array}$ & $\begin{array}{l}\text { Pilot et al. } \\
2006\end{array}$ \\
\hline BIOENV & $\begin{array}{l}\text { Clarke and } \\
\text { Ainsworth } 1993\end{array}$ & $\begin{array}{l}\text { Multivariate, non-para- } \\
\text { metric method that deter- } \\
\text { mines the model that best } \\
\text { explains data of a (dis-) } \\
\text { similarity matrix. }\end{array}$ & $\begin{array}{l}\text { Pair-wise measure of } \\
\text { genetic differentiation } \\
\text { (e.g. pair-wise Fst). }\end{array}$ & $\begin{array}{l}\text { Point-wise measures } \\
\text { (e.g. coordinates or } \\
\text { connectivity indices). }\end{array}$ & $\begin{array}{l}\text { PRIMER } 6 \\
\text { (Primer-E 2006) }\end{array}$ & 9999 permutations & $\begin{array}{l}\text { Spear et al. } \\
2005\end{array}$ \\
\hline
\end{tabular}


can be fitted as co-variates. The confounding effects of these covariates are accounted for before fitting the final model.

\section{Incorporation of genetic data}

For methods requiring a matrix of pair-wise genetic data (i.e. all methods except GESTE, CCA, and BIMR; Table 2), we used pair-wise $\mathrm{F}_{\mathrm{ST}}$ 's (BIOENV, RELATE) or linearized $\mathrm{F}_{\mathrm{ST}}$ (all others; Rousset 1997) to describe genetic differentiation. For CCA, we calculated allele frequencies for each population, following Angers et al. (1999). BIMR and GESTE estimate migration rates and populationspecific $\mathrm{F}_{\mathrm{ST}}$ values directly from genotype data.

\section{Incorporation of landscape data}

When pair-wise landscape data were required, we used distance matrices B, C, and D to describe effective distance among populations. Choosing either untransformed, ln-, or $\log _{10}$-transformed separation distances had little effect (data not shown), and results are based on $\log _{10}$-transformed geographic distances and untransformed resistances.

To measure the presence of landscape boundaries, we created dummy matrices that indicated whether a population pair was separated by a boundary or not (noted in the matrix as 1 or 0 , respectively). For methods working with population-specific landscape measures, we created a dummy variable that placed a population in a certain area not bisected by landscape boundaries (i.e. the population received a " 1 " in the column belonging to that area, and a " 0 " in all other columns).

For several methods (i.e. BIOENV, dbRDA, GESTE, CCA), pair-wise separation distances (i.e. distances $C$ and D) had to be transformed into point-wise measures. This can be achieved through patch- or population-specific connectivity indices often used in meta-population ecology (Moilanen and Nieminen 2002). While most landscape genetic studies utilize pair-wise measures of landscape resistance, population-specific connectivity indices have also been applied in a landscape genetics context (Keyghobadi et al. 2005). Following Foll and Gaggiotti (2006), pair-wise distances were transformed into populationspecific connectivity indices using the formula:

$S_{j}=\sum \exp \left(-\beta d_{i j}\right)$

where $S_{j}$ is the connectivity index for population $j, d_{i j}$ is a measure of pair-wise distance between populations $j$ and $i$ (e.g. geographic distances $\mathrm{B}$, or resistance distances $\mathrm{C}$ or $\mathrm{D}$ ), and the $\beta$ coefficient estimates the distance effect on migration probabilities. The implications of using population-specific vs pair-wise measurements are further elaborated in the discussion section.

\section{Method implementation}

We used all 11 approaches to statistically test for effects of the four landscape variables (i.e. geographic distance B, least-cost distance $\mathrm{C}$, least-cost distance $\mathrm{D}$, landscape boundaries) belonging to a certain study area. Variables were tested for individually (full and partial Mantel test, 
RELATE, full and partial dbRDA) or simultaneously (full and partial CCA, MRDM, BIOENV, BIMR). The GESTE approach is currently limited to a maximum of two landscape variables, so that three analyses were run with this multivariate method: one test for effects of geographic distances, a second test for effects of separation distances $\mathrm{C}$ and $\mathrm{D}$, and a third test for effects of landscape boundaries. Also, one of the scenarios included four areas not bisected by landscape boundaries. Since no more than three distinct areas can be coded for with two variables, this scenario was omitted from the GESTE analyses.

\section{Interpretation of statistical results}

Generally, we interpreted p-values $<0.05$ as a statistically significant influence of a tested landscape variables. For MRDM, we chose a forward, stepwise selection procedure, and a p-value of 0.1 was used to select variables to be included to the model. Variables included into the final model were interpreted as having a significant influence on genetic differentiation. We chose the higher $\mathrm{p}$-value of 0.1 because it is automatically Bonferroni-corrected by the PERMUTE program, so that a p-value of 0.05 would be too conservative. For the BIOENV procedure, variables included in the best model were identified as influential, provided that the best model was significant. For the two Bayesian approaches (GESTE and BIMR), we examined the best supported model to determine important landscape variables. Partial Mantel tests, partial dbRDA, and partial CCA were only applied to data sets in which a significant influence of a landscape variable was detected.

\section{Evaluation of method success}

To test the utility of the various statistical approaches, we calculated power, type-1 error rates, and overall success rates. The power of each method was assessed by calculating the proportion of tests in which the simulated landscapegenetic relationship was correctly detected.

Type-1 error rates indicated how often a method suggested a statistically significant landscape-genetic relationship when no such relationship was simulated. For each method, the proportion of tests that incorrectly detected significant effects was calculated, and the proportion of tests that could be expected to be erroneously significant by chance (i.e. 5\%) was subtracted. Thus, reported error rates are corrected for the number of tests performed with each method, and account for the assumed error level of alpha $=$ 0.05 .

Finally, we calculated overall success rates as the proportion of analyses that led to correct conclusions about simulated landscape-genetic relationships. Analyzing a data set was considered successful if a method indicated a significant effect of the landscape variable used in the simulations, but not of any other, incorrect variables. Thus, overall success rates combine power and corrected type-1 error rates of each method into a single measure of method performance.

We calculated the three measures for each method over all landscape genetic scenarios combined, and separately for each scenario. The partial methods to account for spatial effects are generally not used in isolation, but instead are applied subsequently to full versions of the methods. We therefore estimated the three performance measures for the combination of full and partial method options, and refer to them as Mantel combo, dbRDA combo, and CCA combo, respectively.

\section{Evaluation of method congruence}

To assess the agreement among methods, we used Fleiss' Kappa statistic (Fleiss 1971). This statistic calculates the proportion of cases that are identically classified by two raters, while accounting for rater agreement that could result from chance. For this study, the cases are the different simulated data sets, and the raters are the various statistical approaches. Thus, the Kappa statistic calculates the proportion of data sets that resulted in the same landscape-genetic conclusions with a certain pair of methods. The statistic ranges from $<0$ (no agreement) to 1 (complete agreement), and was interpreted following guidelines given by Landis and Koch (1977). Kappa was calculated over all methods and for all pairs of methods. For methods with a partial option (i.e. Mantel tests, dbRDA, CCA), we only included results for the option with better overall success rates.

\section{Results}

\section{Simulated data}

All pair-wise $\mathrm{F}_{\mathrm{ST}}$ values among populations were significantly different from zero $(\mathrm{p}<0.05)$ in all simulated scenarios and ranged from 0.0114 to 0.1387 . Expected heterozygosity within populations ranged from 0.70 to 0.96 . Geographic distances B and effective distances D were always significantly correlated with each other (Mantel $\mathrm{p}<$ 0.01; range of Mantel r: 0.109 to 0.535), while effective distances $\mathrm{C}$ were not correlated with either $\mathrm{B}$ or $\mathrm{D}$ (Mantel $\mathrm{p}>0.05$ ).

These values demonstrate that our simulations led to a wide range of genetic structure scenarios, and that the landscape variables showed statistical correlations that could readily be encountered in empirical studies. Thus, despite the relative simplicity of our simulations, our study should give an accurate and realistic summary of general method properties for landscape genetic analyses.

\section{Method performance}

Across all landscape genetic scenarios, overall success rates were relatively high for all methods (average 0.76), but varied from 0.59 (standard Mantel tests) to 0.92 (CCA combo; Table 3). Furthermore, the trade-off between power and type- 1 error rates was apparent across all scenarios. While five of the methods had high overall power (i.e. power $>0.94$ ), these methods generally also had high type1 error rates (up to $11 \%$ ). On the other hand, some methods (e.g. GESTE, partial dbRDA) had very low type-1 error rates, but also low power. GESTE did not detect the simulated influence of landscape boundaries (power $=0$ for the E scenario), but performed quite well for the other 
scenarios. When omitting the $\mathrm{E}$ scenario, overall power for GESTE improved to 0.7 , and its overall success rate was slightly above average (0.78).

In summary, the best methods for analyzing the simulated landscape genetic data were (partial) CCA, MRDM, and BIMR (Table 3). These multivariate methods provided a good balance between type-1 error and power, thus leading to correct conclusions for a high percentage of analyzed data sets (overall success rates $\geq 0.87$ ).

When comparing the different landscape genetic scenarios, highest overall success rates were observed for the null scenario (A). For this scenario, most methods accurately concluded that none of the spatial or landscape variables had a significant influence on genetic structures. The standard Mantel test, the Mantel combo, and the RELATE procedure all had very high error rates under the $\mathrm{B}$ scenario (IBD only) of $0.3,0.25$, and 0.22 , respectively. In fact, the standard Mantel test had a success rate of zero for the IBD scenario, because it incorrectly suggested significant influences of additional landscape variables (i.e. in addition to space) in all data sets. The two Mantel approaches also had highest error rates under the $\mathrm{D}$ scenario (landscape resistance/IBD) of 0.21 (Mantel combo) and 0.1 (standard Mantel test), and over all scenarios (0.11 and 0.09 respectively).

BIMR, on the other hand, sometimes identified an erroneous variable, but seldom led to incorrect conclusions when the true variable was identified, thus leading to higher overall success rates (i.e. BIMR suggested the impact of the correct variable, or of an incorrect variable, but not of both in the same data set). The CCA approaches and GESTE were the only methods that had a corrected type- 1 error rate of zero across all scenarios.

\section{Method congruence}

The overall Kappa statistic of 0.506 suggested a moderate level of agreement across all methods (Table 4). Generally, methods that performed well had high levels of agreement with other methods that showed good performance. However, MRDM and the partial Mantel approach had very high agreement according to the Kappa statistic (0.828). This high level of relative agreement is caused by the fact that errors in MRDM always coincided with errors made by the partial Mantel test. Thus, the two methods often had difficulties with the same data sets and can be considered redundant, even though MRDM performed much better than the partial Mantel test. Also, BIMR showed only low to moderate levels of agreement with partial CCA and MRDM, even though these three methods had highest overall success rates. This means that data sets leading to incorrect results in BIMR were not problematic for MRDM or partial CCA.

\section{Discussion}

Our analyses of data sets with known landscape-genetic relationships led to several important findings. First, the most commonly used techniques (i.e. standard Mantel tests and subsequently applied partial Mantel tests) have very 
Table 4. Method congruence assessed through Kappa statistics. The interpretation of statistics is given above the diagonal and follows recommendations of Landis and Koch (1977): Kappa <0: no agreement; 0-0.2: very low agreement; 0.21-0.4: low agreement; 0.61-0.8: substantial agreement, 0.81-1: full agreement. For method abbreviations, see Table 2.

\begin{tabular}{|c|c|c|c|c|c|c|c|c|c|}
\hline & MRDM & CCA combo & BIMR & dbRDA & RELATE & BIOENV & Mantel combo & GESTE & All methods \\
\hline MRDM & - & Substantial & Moderate & Substantial & Substantial & Substantial & Full & Very low & \\
\hline CCA combo & 0.750 & - & Low & Substantial & Moderate & Substantial & Substantial & Very low & \\
\hline BIMR & 0.425 & 0.409 & - & Low & Low & Moderate & Moderate & Low & Kappa: \\
\hline $\mathrm{dbRDA}$ & 0.629 & 0.635 & 0.400 & - & & Moderate & Moderate & Very low & 0.506 \\
\hline RELATE & 0.678 & 0.511 & 0.319 & 0.412 & - & Moderate & Substantial & Very low & (Moderate \\
\hline BIOENV & 0.680 & 0.746 & 0.411 & 0.676 & 0.456 & - & Substantial & Moderate & agreement) \\
\hline Mantel combo & 0.828 & 0.687 & 0.413 & 0.555 & 0.641 & 0.668 & - & Very low & \\
\hline GESTE & 0.125 & 0.131 & 0.220 & 0.118 & 0.181 & 0.430 & 0.152 & - & \\
\hline
\end{tabular}

high type-1 error rates. Errors were caused by the methods' inability to distinguish between landscape effects that actually influenced genetic structure and other correlated distances. Thus, with our simulated data, the Mantel approaches often suggested the importance of landscape variables that do not really influence gene flow. This illustrates the challenge of distinguishing between spatial effects (i.e. pure IBD) and true landscape effects with the Mantel tests. Furthermore, standard Mantel tests based on landscape variables not used in the simulations often led to slightly higher correlation coefficients than tests based on the correct variable. Thus, assessing the relative importance of landscape variables by comparing the strength of raw r-values obtained from multiple standard Mantel tests is likely inappropriate for landscape genetic purposes.

BIMR also had a high type- 1 error rate under the $B$ scenario (0.1), but had better overall success rates than the two Mantel approaches. This difference is caused by the independent calculations of power, error and success rates: while Mantel tests always identified effective distance B as being influential (power $=1$ ), they also often suggested a significant effect of additional variables, thus having low overall success rates.

Oftentimes, studies test for the influence of more than one separation distance among sampling localities (Spear et al. 2005, Cushman et al. 2006, Epps et al. 2007), but multicollinearity among these separation distances is seldom considered. Whether different measures of population separation are correlated or not will depend on the landscape, and on the exact approach to measure interpopulation distances (e.g. least cost distances, length of least-cost path, currents derived from circuit theory; McRae 2006). We recommend that researchers report correlations among all distance matrices utilized in their studies, and carefully evaluate whether multicollinearity could be problematic for Mantel approaches. In fact, even when correlations among different separation distances are insignificant, results obtained from the Mantel approaches should be interpreted with caution. The Mantel tests are insensitive to non-linear relationships, and the usefulness of the partial Mantel test for genetic studies has been questioned (Raufaste and Rousset 2001, Castellano and Balletto 2002, Rousset 2002). In sum, there are few reasons for using Mantel approaches in landscape genetics, except that these tests are easy to conduct and interpret. Thus, Mantel tests may be most appropriate for preliminary or exploratory analyses in the early stages of landscape genetic research projects. Such exploratory analyses are neglected in current landscape genetic studies, and little attention is given to data distributions and response curves. We visually inspected the distribution of our data, and the shape of the landscape-genetic relationships. Many of the data sets showed multi-modal and skewed distributions, and using linearized $\mathrm{F}_{\mathrm{ST}}$ did not always lead to truly linear response curves. While we did not detect any trends in method performances with respect to data distributions and response curves, this may be due to the relatively small sample sizes. Thus, researchers should evaluate and report the characteristics of their data, and future studies should evaluate how sensitive various methods are to violations of underlying assumptions, such as data normality and linearity. Also, the utility of more advanced statistical approaches related to the Mantel statistic (e.g. Mantel correlograms; Smouse et al. 1986) needs to be investigated separately.

The multivariate methods implemented in PERMUTE (for multiple regression on distance matrices) and BIMR were more successful in detecting the simulated landscapegenetic relationships. PERMUTE uses a step-wise procedure to infer the influences of predictor variables, and this approach worked well with our simulated data, which had only few populations, few predictor variables, and very clear landscape-genetic relationships. However, step-wise approaches have been criticized for biased and inconsistent results (Whittingham et al. 2006), and the performance of stepwise MRDM with larger, more complex data sets (e.g. for individual-level analyses) needs additional evaluation. Also, MRDM cannot directly account for possible variable interactions, and assumes a strictly linear relationship between dependent and independent variables. The BIMR method, on the other hand, relaxes this assumption through the use of a GLM, and can include variable interactions in the model. Thus, this approach should be suitable for analyzing very complex empirical data sets, but it can only be used for population-level analyses.

CCA can be adapted for individual-level analyses, for example by coding alleles as absent (0), in heterozygote state (1), or homozygote state (2) (Cushman et al. 2006). The (partial) CCA performed very well in this study, despite the fact that it is not designed for explanatory data measured between sampling localities, so that pair-wise distances had to be transformed into population-specific connectivity indices. Results suggest that these local connectivity indices can be use as an alternative to pair-wise measures, even though these indices essentially summarize the variation of the data, and often require additional parameters (e.g. 
dispersal distance) to be estimated. The discussion of how to quantify connectivity is ongoing, and our study should not be interpreted as favoring one approach over the other. Expressing landscape data through population-specific measurements focuses on processes operating at sampling locations, while pair-wise measurements emphasize inbetween processes. Thus, in-between measures describe connectivity as an attribute of the landscape, while local indices describe it as an attribute of an individual patch or population (Fischer and Lindenmayer 2007). In reality, landscape variables both at and in-between sampling sites can influence gene flow. For example, population sizes and density-dependent emigration rates can be influenced by the size and quality of a patch, while the fate of emigrants is influenced by the matrix among patches (Fahrig 2007). Thus, connectivity indices may not be appropriate and effective for all data sets, but certainly have potential for landscape genetics, because they can include additional information such as patch or population sizes, habitat quality or carrying capacities (Saura and Pascual-Hortal 2007). Emerging graph-theoretic approaches (Urban and Keith 2001) could also greatly contribute to the development of meaningful connectivity measures for landscape genetics. Overall, both patch-specific and pair-wise measurements have advantages and limitations, and how to quantify effective separation distances for landscape genetics should depend on the exact research questions and predictions.

Partial CCA was most effective in accounting for spatial effects (i.e. IBD) through the incorporation of coordinates as covariates. Also, the method can be integrated with geostatistics to distinguish between spatial autocorrelation and spatial dependence (Wagner 2004). Furthermore, CCA was specifically designed for very large, complex data sets, and it can be adjusted for non-linear response curves (Makarenkov and Legendre 2002). Overall, CCA has great potential to be an effective and highly flexible technique in landscape genetics, and we encourage scientists to explore its utility in more detail.

\section{Study limitations}

Our simulation-based study was designed to identify advantages and limitations of statistical approaches used in landscape genetics. However, simulations always involve assumptions and simplifications that need to be considered when interpreting results. For all our simulations, the number of sampled populations, number of individuals per populations, sex ratio, and number of loci were held constant. We also did not incorporate any historic landscape influences, asymmetrical gene flow patterns, nonequilibrium conditions, fluctuating population sizes or (meta-)population dynamics into our simulations. Finally, we focused on neutral genetic structure among populations as one component of genetic variation (Lowe et al. 2004, Holderegger et al. 2006).

Partially, our simulation approach was limited by the lack of landscape genetic simulation software. However, our goal was to obtain a general overview of method performance across a diversity of well-established gene flow scenarios. While our simulations do not include all possible complex- ities of empirical data, the simulated scenarios encompass a wide range of landscape-genetic relationship found in empirical studies. This resulted in data sets with feasible and clear landscape-genetic relationships, making it possible to compare the different methods in an intuitive framework.

In addition to these simulation-based limitations, the comparison of results was challenging due to the large variety of statistical approaches. Most of the methods we evaluated were not developed for genetic data, and those that were are still difficult to compare directly. For example, GESTE estimates population-specific indices that describe genetic structure among subpopulations of a larger metapopulation system. BIMR, on the other hand, estimates recent migration rates among sampling areas, and identifies environmental factors that influence these rates. Thus, while the two methods may seem computationally similar, they actually have quite different goals. Nevertheless, all of the methods can be used to statistically test for landscapegenetic relationships, and they can be compared based on this commonality. To do so, our objective was to make the interpretation of results as simple and fair as possible, but our approach may have favored certain techniques over others. For example, the two Bayesian approaches (BIMR and GESTE) were used in a hypothesis-testing framework, which is obviously in contradiction to Bayesian theory. Furthermore, Foll and Gagiotti (2006) cautioned that while Bayesian approaches can be highly effective, they also require a good understanding of the underlying theory, and careful evaluation of model performance. However, results in both Bayesian methods were often very clear, with a single model identified as most likely by percentages $>60 \%$. Thus, even without strict hypothesis testing, researchers probably would have come to the same landscape-genetic conclusions. While BIMR performed well with our data, the GESTE program could not detect the influences of landscape boundaries, possibly because the analysis of this scenario involved categorical predictor variables. Considering that GESTE showed good performance in a previous evaluation involving categorical data (Foll and Gaggiotti 2006), it is possible that our coding of landscape boundaries through dummy variables, or the chosen MCMC parameter settings were not optimal. Furthermore, GESTE, CCA, BIOENV, and dbRDA were not designed for landscape data measured between sampling locations, which could explain the generally lower power of these methods. Finally, dbRDA is also available in a stepwise modeling framework (Pilot et al. 2006), which may be more appropriate for landscape genetic data analysis. Despite these limitations, the analysis of the simulated data was valuable for comparing and evaluating current statistical approaches used in landscape genetics. We encourage others to expand this work by evaluating new methods and more complex scenarios. For example, the impact of varying simulation parameters, number of loci, and number of individuals sampled per population could be evaluated through sensitivity analyses. Similarly, our study did not evaluate the sensitivity of methods to imperfectly or incorrectly defined cost values for the calculation of landscape resistances. The influences of landscape composition and configuration on landscape genetic analyses can also not be evaluated from our study. We simulated data sets similar to those found in empirical studies, by basing our 
analyses on readily available GAP data. A different approach could consist of simulating neutral landscapes, in which configuration and composition can be controlled. By repeatedly simulating similar landscapes for the subsequent genetic simulations, it would be possible to determine which methods work best for landscapes showing certain characteristics. Finally, we focused on population-level analyses, but an increasing number of studies analyzes genetic patterns among individuals (Manel et al. 2003, Coulon et al. 2004, Cushman et al. 2006). Except for GESTE and BIMR, all of the tested methods can theoretically be used with individual-level data, but this does not mean that they are all suitable for individual-based analyses. For example, methods that require locationspecific connectivity indices are difficult to apply to individuals, because connectivity indices are intended to describe connectivity among populations or patches. Furthermore, sample sizes for individual-level analyses are often much higher than for population-level analyses, and software available for some of the tested methods may simply not be able to handle such large data sets (e.g. PERMUTE for stepwise regression).

Overall, many additional research possibilities remain, and emerging simulation software (e.g. EcoGenetics, $<$ www2.unil.ch/biomapper/ecogenetics/index.html >) will make such evaluations easier and more accessible.

\section{Suggestions for future analytical improvement}

In addition to evaluation of existing methods, developing improved techniques is necessary to advance landscape genetic analyses. The four most successful approaches are all multivariate, and three of them are not (strictly) linear. When omitting the E scenario (landscape boundaries), GESTE is a fifth multivariate, not strictly linear method with better-than-average performance. We therefore encourage researchers to apply and develop other statistical approaches that are multivariate and non-linear. For example, generalized dissimilarity modeling (GDM) is a non-linear extension of matrix regression, and can directly be applied to a pair-wise matrix of (dis-)similarities (Ferrier et al. 2007). Thus, this method could potentially be useful for landscape genetics, but it has not been applied in this context.

Furthermore, future analytical development should focus on methods that are non-linear, multivariate (including variable interactions), and provide options for effectively separating spatial autocorrelation from true landscape effects. Finally, incorporating both at and in-between landscape variables into a single analysis may be beneficial for some data sets. Gravity-type models can use both kinds of data, and they are currently introduced into landscape genetics by Murphy (2008).

In addition to finding and developing effective statistical methods, researchers also need to further develop the general analytical framework used in landscape genetics. Currently, most landscape genetic analyses are limited to a simple hypothesis testing approach, and we have followed this approach in our simulation study. However, this approach implicitly assumes that the null-hypothesis is known and true, and it only separates "important" (i.e. statistically significant) and "not important" (i.e. statistically insignificant) landscape variables, without evaluating their relative biological importance. Most current studies also do not state their null- and alternative hypotheses a priori, and simple hypothesis testing is likely ineffective for analyzing very complex relationships.

Based on the relatively high type- 1 errors shown in this study, researchers should report what landscape-genetic mechanisms they are testing for, instead of trying to find reasonable explanations for observed relationships a posteriori. Sometimes, good expectations for landscape-genetic relationships are difficult to derive, because little is known about a study organism. In such cases, data mining approaches (e.g. RandomForests; Breiman 2001, Murphy 2008) could be used to detect important patterns. However, in most instances, some data on species biology and landscape ecology exist, and these data should be used to develop valid and feasible predictions about how the landscape is influencing genetic variation. Conceptual flowcharts and graphs (e.g. cascading graph diagrams; Aarssen 2004) can facilitate this process, by simplifying and structuring assumed causal relationships between landscape variables and genetic patterns. Identifying the actual biological and ecological mechanisms that link landscape heterogeneity to genetic variation will greatly improve landscape genetic inferences, and lead to a better understanding of genetic processes in real landscapes.

Except for the two Bayesian approaches, current methods add little to our understanding of relative effects of various landscape variables. Many alternatives to classical hypothesis-testing exist (Stephens et al. 2007), and landscape geneticists should evaluate the potential of these approaches for the analysis of their data. For example, information-theoretic approaches (Burnham and Anderson 2002) can be useful in finding factors influencing genetic data (Yang 2004, Spear et al. 2005), and could help to develop predictive landscape genetic models. However, landscape genetic data is often pair-wise, and future research needs to determine whether certain information-theoretic approaches can be used for this kind of data. Appropriately identified models could be used to predict genetic consequences of various climate and landscape change scenarios, and thus would be an important contribution to ongoing research efforts. Since most landscape genetic data will show spatial autocorrelation, modeling techniques that explicitly account for, and make use of, spatial autocorrelation (Lichstein et al. 2002, Epperson 2003) are particularly promising.

\section{Conclusions and recommendations for current studies}

Results obtained in this study suggest that no single, optimal method currently exists for landscape genetic analyses, but certain methods clearly performed better than others in our simulated scenarios. Also, results show only moderate agreement among methods, so that different analytical choices could lead to varying and potentially erroneous conclusions. On the other hand, some methods showed very high levels of agreement, and are therefore redundant. Based on these results, we recommend that 
researchers apply multiple, non-redundant statistical approaches for landscape genetic data analysis. Specifically, combinations of BIMR and MRDM appear effective when landscape effects between sampling location are of interest. When the focus is on population-specific landscape effects, (partial) CCA and GESTE can be combined, though the use of the latter with categorical data warrants further investigation. Considering the highly complex nature of landscape genetics and the large variety of landscape genetic research questions, it is unlikely that a single method will ever suit all research needs. Using multiple methods to analyze the same data will lead to increased certainty of inferences, and ensures that conclusions drawn from landscape genetic analyses are valid.

In conclusion, much additional research is needed in method development and validation, and for deriving landscape genetic theory that is truly based on landscapeecological principles. For now, recommendations based on this study will hopefully make landscape genetics studies more comparable and reliable, and contribute to the future development of this promising field.

Acknowledgements - We greatly appreciate the valuable comments on this manuscript provided by Melanie Murphy, Jeff Evans and members of the Waits lab group. Comments from three anonymous reviewers further improved the manuscript. Funding for this research was provided by the German-American Fulbright program, the Univ. of Idaho (UI) Center for Research on Invasive Species and Small Populations (CRISSP), the UI College of Graduate Studies, and grant No. PS07N003 from the UI Student Grant Program.

\section{References}

Aarssen, L. W. 2004. Interpreting co-variation in species richness and productivity in terrestrial vegetation: making sense of causations and correlations at multiple scales. - Folia Geobot. 39: 385-403.

Adriaensen, F. et al. 2003. The application of 'least-cost' modelling as a functional landscape model. - Landscape Urban Plan. 64: 233-247.

Angers, B. et al. 1999. Canonical correspondence analysis for estimating spatial and environmental effects on microsatellite diversity in brook char (Salvalinus fontinalus). - Mol. Ecol. 8: 1043-1053.

Balloux, F. 2002. Easypop version 1.8. A software for population genetics simulations. - Univ. of Edinburgh.

Blank, J. et al. 2007. Habitat type does not affect population genetic structure in sympatric great tits (Parus major) and blue tits (P. caerules). - J. Negative Results 4: 1-14.

Bonnet, E. and Van de Peer, Y. 2002. ZT: a software tool for simple and partial Mantel tests. - Ghent Univ.

Borg, I. and Groenen, P. 2005. Modern multidimensional scaling: theory and applications. - Springer.

Breiman, L. 2001. Random forests. - Mach. Learn. 45: 5-32.

Burnham, K. and Anderson, D. 2002. Model selection and multimodel inference: a practical information-theoretic approach. - Springer.

Castellano, S. and Balletto, E. 2002. Is the partial Mantel test inadequate? - Evolution 56: 1871-1873.

Clarke, K. and Ainsworth, M. 1993. A method of linking multivariate community structure to environmental variables. - Mar. Ecol. Prog. Ser. 92: 205-219.
Clarke, K. and Warwick, R. 2001. Change in marine communities: an approach to statistical analysis and interpretation. - PRIMER-E, Plymouth.

Coulon, A. et al. 2004. Landscape connectivity influences gene flow in a roe deer population inhabiting a fragmented landscape: an individual-based approach. - Mol. Ecol. 13: 2841.

Cushman, S. A. et al. 2006. Gene flow in complex landscapes: testing multiple hypotheses with causal modeling. - Am. Nat. 168: 486-499.

Epperson, K. 2003. Geographical genetics. - Princeton Univ. Press.

Epps, C. et al. 2005. Highways block gene flow and cause rapid decline in genetic diversity of desert bighorn sheep. - Ecol. Lett. 8: 1029-1038.

Epps, C. et al. 2007. Optimizing dispersal and corridor models using landscape genetics. - J. Appl. Ecol. 44: 714-724.

Fahrig, L. 2007. Landscape heterogeneity and metapopulation dynamics. - In: Wu, J. and Hobbs, R. J. (eds), Key topics and perspectives in landscape ecology. Cambridge Univ. Press, pp. 78-89.

Faubet, P. and Gaggiotti, O. E. 2008. A new Bayesian method to identify the environmental factors that influence recent migration. - Genetics 178: 1491-1504.

Ferrier, S. et al. 2007. Using generalized dissimilarity modelling to analyse and predict patterns of beta diversity in regional biodiversity assessment. - Divers. Distrib. 13: 252-264.

Fischer, J. and Lindenmayer, D. B. 2007. Landscape modification and habitat fragmentation: a synthesis. - Global Ecol. Biogeogr. 16: 265-280.

Fleiss, J. L. 1971. Measuring nominal scale agreement among many raters. - Psychol. Bull. 86: 974-977.

Foll, M. and Gaggiotti, O. E. 2006. Identifying the environmental factors that determine the genetic structure of populations. - Genetics 174: 875-891.

Goudet, J. 1995. FSTAT (version 1.2): a computer program to calculate F-statistics. - J. Hered. 86: 485-486.

Holderegger, R. and Wagner, H. H. 2006. A brief guide to landscape genetics. - Landscape Ecol. 21: 793-796.

Holderegger, R. and Wagner, H. H. 2008. Landscape genetics. - BioSciences 58: 199-207.

Holderegger, R. et al. 2006. Adaptive vs neutral genetic diversity: implications for landscape genetics. - Landscape Ecol. 21: 797-807.

Holzhauer, S. I. J. et al. 2006. Effect of historic landscape change on the genetic structure of the bush-cricket Metrioptera roeseli. - Landscape Ecol. 21: 891-899.

Keyghobadi, N. et al. 2005. Among- and within-patch components of genetic diversity respond at different rates to habitat fragmentation: am empirical demonstration. - Proc. R. Soc. B 272: 553-560.

Landis, J. R. and Koch, G. G. 1977. The measurement of observer agreement for categorical data. - Biometrics 33: 159-174.

Leclerc, E. et al. 2008. The landscape genetics of yellow perch (Perca flavescens) in a large fluvial ecosystem. - Mol. Ecol. 17: 1702-1717.

Legendre, P. 1993. Spatial autocorrelation: trouble or new paradigm? - Ecology 74: 1659-1673.

Legendre, P. 2000. Comparison of permutation methods for the partial correlations and partial Mantel tests. - J. Stat. Comput. Sim. 67: 37-73.

Legendre, P. and Legendre, P. 1998. Numerical ecology. - Elsevier.

Legendre, P. and Anderson, M. J. 1999. Distance-based redundancy analysis: testing multispecies responses in multifactorial ecological experiments. - Ecol. Monogr. 69: 1-24. 
Legendre, P. et al. 1994. Modeling brain evolution from behavior: a permutational regression approach. - Evolution 48: $1487-1499$.

Lichstein, J. W. et al. 2002. Spatial autocorrelation and autoregressive models in ecology. - Ecol. Monogr. 72: 445-463.

Lowe, A. et al. 2004. Ecological genetics. - Blackwell.

Makarenkov, V. and Legendre, P. 2002. Nonlinear redundancy analysis and canonical correspondence analysis based on polynomial regression. - Ecology 83: 1146-1161.

Manel, S. et al. 2003. Landscape genetics: combining landscape ecology and population genetics. - Trends Ecol. Evol. 18: 189-197.

Manly, B. F. J. 1986. Randomization and regression methods for testing for associations with geographical, environmental and biological distances between populations. - Res. Popul. Ecol. 28: 201-218.

Manly, B. F. J. 2007. Randomization, bootstrap and Monte Carlo methods in biology. - Chapman and Hall/CRC.

Mantel, N. 1967. The detection of disease clustering and a generalized regression approach. - Cancer Res. 27: 209-220.

McArdle, B. and Anderson, M. 2001. Fitting multivariate models to community data: a comment on distance-based redundancy analysis. - Ecology 82: 290-297.

McRae, B. H. 2006. Isolation by resistance. - Evolution 60: 1551-1561.

Moilanen, A. and Nieminen, M. 2002. Simple connectivity measures in spatial ecology. - Ecology 83: 1131-1145.

Murphy, M. A. 2008. New approaches in landscape genetics and niche modeling for understanding limits to anuran distributions. - Ph.D. thesis, Washington State Univ.

Pilot, M. et al. 2006. Ecological factors influence population genetic structure of European grey wolves. - Mol. Ecol. 15: 4533-4553.

Primer-E 2006. PRIMER 6, version 6.1.6. - Primer-E, Plymouth, UK.

Raufaste, N. and Rousset, F. 2001. Are partial Mantel tests adequate? - Evolution 55: 1703-1705.

Ray, N. 2005. PATHMATRIX: a GIS tool to compute effective distances among samples. - Mol. Ecol. Not. 5: 177-180.

Raymond, M. and Rousset, F. 1995. GENEPOP (version 1.2): population genetics software for exact tests and ecumenicism. - J. Hered. 86: 248-249.

Ricketts, T. H. 2001. The matrix matters: effective isolation in fragmented landscapes. - Am. Nat. 158: 87-99.

Rose, C. G. et al. 2006. Isolation by distance in the eastern oyster, Crassostrea virginica, in Chesapeake bay. - J. Hered. 97: 158-170.

Download the Supplementary material as file E5807 from $<$ www.oikos.ekol.lu.se/appendix $>$.
Rousset, F. 1997. Genetic differentiation and estimation of gene flow from F-statistics under isolation by distance. - Genetics 145: $1219-1228$.

Rousset, F. 2002. Partial Mantel tests: reply to Castellano and Balletto. - Evolution 56: 1874-1875.

Sacks, B. N. et al. 2004. Population structure of California coyotes corresponds to habitat-specific breaks and illuminates species history. - Mol. Ecol. 13: 1265-1275.

Saura, S. and Pascual-Hortal, L. 2007. A new habitat availability index to integrate connectivity in landscape conservation planning: comparison with existing indices and application to a case study. - Landscape Urban Plan. 83: 91-103.

Smouse, P. E. et al. 1986. Multiple regression and correlation extensions of the Mantel test of matrix correspondence. - Syst. Zool. 35: 627-632.

Spear, S. F. et al. 2005. Landscape genetics of the blotched tiger salamander (Ambystoma tigrinum melanostictum). - Mol. Ecol. 14: 2553-2564.

Stephens, P. A. et al. 2007. Inference in ecology and evolution. - Trends Ecol. Evol. 22: 192-197.

Storfer, A. et al. 2007. Putting the "landscape" in landscape genetics. - Heredity 98: 128-142.

Systat Software 2004. Systat 11.0. - Systat Software.

ter Braak, C. 1986. Canonical correspondence analysis: a new eigenvector technique for multivariate direct gradient analysis. - Ecology 67: 1167-1179.

ter Braak, C. and Smilauer, P. 2006. Canoco for Windows version 4.54. - Biomotris-Plant Research.

Trizio, I. et al. 2005. Geographical distance and physical barriers shape the genetic structure of Eurasian red squirrels (Sciurus vulgaris) in the Italian Alps. - Mol. Ecol. 14: 469-481.

Urban, D. and Keith, T. 2001. Landscape connectivity: a graphtheoretic perspective. - Ecology 82: 1205-1218.

Wagner, H. H. 2004. Direct mutli-scale ordination with canonical correspondence analysis. - Ecology 85: 342-351.

Whittingham, M. J. et al. 2006. Why do we still use stepwise modeling in ecology and behavior? - J. Anim. Ecol. 75: 1182-1189.

Wright, S. 1943. Isolation by distance. - Genetics 28: 114-138.

Wright, S. 1977. Evolution and the genetics of populations. - Chicago Press.

Yang, R.-C. 2004. A likelihood-based approach to estimating and testing for isolation by distance. - Evolution 58: 1839-1845. 